\title{
29. ALGUNOS PTERIDÓFITOS DEL SUR DE ESPAÑA
}

José LARA RUIZ

Palabras clave: Pteridófitos, corología, España.

Se presentan varias citas de interés biogeográfico para la Pteridoflora del Sur de España. Para cada especie se da la cuadrícula UTM de $10 \mathrm{Km}$ de lado. Los pliegos testimonio se encuentran depositados en el Herbario MGC.

Asplenium celtibericum Rivas Martínez

GRANADA: Sierra de Castril, Castril, 1800 m., rupícola. WG 18. VII/1991. J. Lara Ruiz (MGC 32884).

Asplenium fontanum (L.) Bernh.

ALMERIA: Sierra de María, El Mojonar, 1600 m., rupícola. WG66. 20/VII/1991. J. Lara Ruiz (MGC 32741).

JAEN: Sierra de Cazorla, Sierra del Pozo, Pozo Alcón, hacia el pico Cabañas, 1600 m., rupícola. WG08. 14/VII/1991. J. Lara Ruiz (MGC 32742).

Asplenium onopteris $\mathrm{L}$.

MURCIA: Sierra del Gigante, Fontanares, matorral, 950 m. WG 97. VII/1991. J. Lara Ruiz (MGC 32733).

Asplenium viride Huds.

JAEN: Sierra de Cazorla, Sierra de las Cuatro Villas, Iznatoraf, La Muela Alta, 1400 m., rupícola. WH01. VII/1991. J. Lara Ruiz (MGC 32882).

Cheilanthes hispanica Mett.

GRANADA: Sierra de Castril, Castril, sobre el pueblo, 1000 m., rocas ácidas. WG18. VII/1991. J. Lara Ruiz (MGC 32881).

Cryptogramma crispa (L.) R. Br. ex Hook.

JAEN: Sierra del Segura, Santiago de la España, base de Los Puestos, 1700 m., rupícola. WH32. VII/1991. J. Lara Ruiz (MGC 32880).

Dryopteris affinis (Lowe) Fraser-Jenkins subsp. borreri (Newman) Fraser-Jenkins

ALMERIA: Sierra de María, María, sobre el Cortijillo, matorral. 1500 m., WG76. VIII/1991. J. Lara Ruiz (MGC 32883).

Equisetum arvense $\mathrm{L}$.

MURCIA: Sierra del Gigante, Fontanares, 900 m. sitios húmedos. WG97. VII/1991. J. Lara Ruiz (MGC 32744).

Ophioglossum lusitanicum L.

JAEN: Sierra de Cazorla, Sierra de las Cuatro Villas, Villacarrillo, Huerta del Cura, 500 m., prado ácido encharcado. WH01. VII/1991. J. Lara Ruiz (MGC 32877).

Ophioglossum vulgatum $\mathrm{L}$.

ALMERIA: Sierra de María, Maria, sobre el Cortijillo, 1500 m., prado húmedo. WG76. VII/1991. J. Lara Ruiz (MGC 32879).

Polystichum aculeatum (L.) Roth

ALMERIA: Sierra de María, El Mojonar, matorral húmedo. 1100 m. WG66. 20/VII/ 1991. J. Lara Ruiz (MGC 32740).

Polystichum lonchitis (L.) Roth

JAEN: Sierra de Segura, Hornos de Segura, rupícola. 1000 m. WH23. 30/VII/1990. J. Lara Ruiz (MGC 32739).

(Aceptado para su publicación en Abril de 1992)

Dirección del autor: Departamento de Biología Vegetal. Facultad de Biología 08028, Barcelona. 